
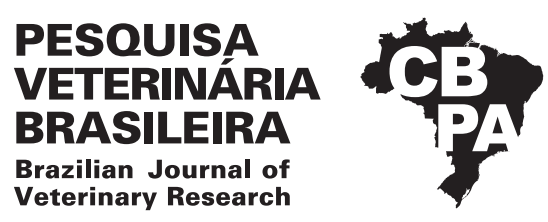

Pesq. Vet. Bras. 38(8):1656-1663, agosto 2018 DOI: 10.1590/1678-5150-PVB-5451

Artigo Original

ISSN 0100-736X (Print)

ISSN 1678-5150 (Online)

\title{
Fisioterapia na recuperação funcional e qualidade de vida de cães paraplégicos por doença do disco intervertebral (Hansen tipo I) toracolombar submetidos à cirurgia descompressiva ${ }^{1}$
}

\author{
Amanda O. Andrades², Angel Ripplinger², Dênis A. Ferrarin², Graciane Aiello², \\ Luciana Schneider ${ }^{2}$, Marcelo L. Schwab ${ }^{2}$ e Alexandre Mazzanti*
}

\begin{abstract}
Andrades A.O., Ripplinger A., Ferrarin D.A., Aiello G., Schneider L., Schwab M.L. \& Mazzanti A. 2018. [Physiotherapy in functional recovery and quality of life of paraplegic dogs due to thoracolumbar intervertebral disc disease (Hansen type I) submitted to decompressive surgery.] Fisioterapia na recuperação funcional e qualidade de vida de cães paraplégicos por doença do disco intervertebral (Hansen tipo I) toracolombar submetidos à cirurgia descompressiva. Pesquisa Veterinária Brasileira 38(8):1656-1663. Laboratório de Fisioterapia Veterinária, Departamento de Pequenos Animais, Centro de Ciências Rurais, Universidade Federal de Santa Maria, Avenida Roraima 1000, Camobi, Santa Maria, RS 97105-900, Brazil. E-mail: alexamazza@yahoo.com.br

The retrospective study included paraplegic dogs affected by thoracolumbar intervertebral disc disease with absence of deep pain perception and submitted to a decompressive surgical technique. The aim was to compare functional recovery and quality of life (QOL) of dogs that underwent a home physiotherapy protocol to dogs that were cared in a specialized center. Twenty-eight dogs were placed in group A (GA, home physiotherapy protocol) and other 38 patients were placed in group B (GB, physiotherapy protocol in a specialized center). Forty three percent (43\%) of the patients recovered the ability to walk in an average of 40 postoperative (PO) days in GA and $42 \%$ in an average of 36 days in GB. In $53 \%$ of the cases $(35 / 66)$ the dogs remained paraplegic without deep pain perception at the end of physiotherapeutic protocols. Twenty-five owners from GA and 26 from GB answered a questionnaire about QOL, with a minimum of six PO months. The evaluation of the animals survival time, euthanasia and the QOL score demonstrated that dogs that underwent a physiotherapeutic treatment in a specialized center probably were benefited by the close monitoring and orientation to owners until 90 days of $\mathrm{PO}$.
\end{abstract}

INDEX TERMS: Physiotherapy, recovery, paraplegia, dogs, intervertebral disc disease, Hansen type I, rehabilitation, neurology, surgery.

RESUMO.- Este estudo retrospectivo incluiu cães paraplégicos com ausência de percepção a dor profunda secundário a doença do disco intervertebral toracolombar e submetidos à cirurgia descompressiva. Teve como objetivo comparar a

\footnotetext{
${ }^{1}$ Recebido em 20 de maio de 2017.

Aceito para publicação em 28 de junho de 2017.

${ }^{2}$ Programa de Pós-Graduação em Medicina Veterinária, área de concentração em Clínica e Cirurgia Veterinária, Centro de Ciências Rurais (CCR), Universidade Federal de Santa Maria (UFSM), Av. Roraima 1000, Camobi, Santa Maria, RS 97105-900, Brasil.

${ }^{3}$ Laboratório de Fisioterapia Veterinária (Fisiovet), Departamento de Clínica de Pequenos Animais, Centro de Ciências Rurais (CCR), Universidade Federal de Santa Maria (UFSM), Av. Roraima 1000, Camobi, Santa Maria, RS 97105-900. *Autor para correspondência: alexamazza@yahoo.com.br
}

recuperação funcional e a qualidade de vida (QV) quando submetidos a um protocolo em um centro especializado ou a domicílio realizado pelo tutor. Vinte e oito cães foram distribuídos no grupo A (GA) e submetidos ao protocolo domiciliar e 38 denominados grupo B (GB) em um centro especializado. Recuperaram a habilidade de caminhar $43 \%$ dos cães em uma média de 40 dias de pós-operatório (PO) no GA e $42 \%$ em uma média de 36 dias no GB. Em 53\% dos casos (35/66) os cães permaneceram paraplégicos sem percepção a dor profunda ao final do período de 90 dias de PO. Vinte e cinco tutores do GA e 26 do GB responderam um questionário sobre a QV, com no mínimo seis meses de PO. A avaliação do tempo de sobrevida dos animais, realização 
de eutanásia e nota atribuída a QV demonstraram que os cães do GB provavelmente foram beneficiados pelo estreito acompanhamento e orientação aos tutores até 90 dias PO.

TERMOS DE INDEXAÇÃO: Fisioterapia, recuperação, caninos, paraplegia, doença do disco intervertebral, Hansen tipo I, reabilitação, neurologia, cirurgia.

\section{INTRODUÇÃO}

A extrusão do disco intervertebral é uma das causas mais comuns de alterações neurológicas em cães, sendo provocada pela degeneração do disco intervertebral (Brisson 2010, Fingeroth \& Thomas 2015). A região toracolombar, particularmente entre T11-L2, é a mais afetada e os sinais clínicos são atribuídos à lesão causada pelo impacto (contusão) e/ou à compressão mecânica na medula espinhal e/ou raízes nervosas. Podem variar desde hiperestesia espinhal até paraplegia com perda da percepção a dor profunda ou nocicepção (Kazakos et al. 2005).

Cães paraplégicos são considerados emergência cirúrgica (Dewey \& Costa 2016) e encontram-se variações nas taxas e tempo de recuperação funcional para àqueles com ausência de dor profunda (Scott \& McKee 1999, Olby et al. 2003, Kazakos et al. 2005, Laitinen \& Puerto 2005, Arias et al. 2007, Festugatto et al. 2008, Muguet-Chanoit et al. 2012, Santos et al. 2012, Jeffery et al. 2016), este sinal considerado um indicador de prognóstico (Brisson 2010, Granger \& Carwardine 2014, Fingeroth \& Thomas 2015, Dewey \& Costa 2016), que se torna ainda mais desfavorável quando não a recuperam dentro de duas a quatro semanas após a descompressão medular (Brisson 2010).

A prática da fisioterapia é uma das recentes adições ao protocolo terapêutico de pacientes com doença do disco intervertebral (DDIV) e tem sido recomendada a fim de auxiliar na recuperação após a descompressão cirúrgica (Wall 2015). Os principais objetivos são recuperar ou manter a amplitude do movimento articular, minimizar a atrofia muscular e prevenir ou amenizar o desconforto do paciente, resultando em períodos mais curtos de internação e melhorando o seu bem-estar (Dewey \& Costa 2016).

Quando não ocorria rápida reversão das deficiências provocadas pela lesão da medula espinhal de forma espontânea ou em resposta a descompressão cirúrgica, os cães, muitas vezes, eram submetidos à eutanásia. Com isso, a fisioterapia pode, de forma adjuvante, oferecer maiores chances de recuperação e proporcionar boa qualidade de vida (QV) aos pacientes com DDIV (Wall 2015).

Uma das limitações do emprego da fisioterapia está na dificuldade de deslocamento semanais dos pacientes até os centros especializados. Assim, há necessidade da orientação pelo fisioterapeuta veterinário da elaboração de um protocolo fisioterapêutico que possa ser realizado a domicilio, buscando auxiliar na recuperação ou de amenizar as sequelas de desuso.

Diante disso, o objetivo deste estudo retrospectivo foi comparar a recuperação funcional e a QV de cães paraplégicos sem percepção a dor profunda, acometidos de DDIV toracolombar, após a descompressão cirúrgica e submetidos a um protocolo de fisioterapia em um centro especializado ou domiciliar.

\section{MATERIAL E MÉTODOS}

Os arquivos do Laboratório de Fisioterapia Veterinária (Fisiovet) de uma Instituição de ensino superior foram revisados entre abril de 2008 e junho de 2016, em busca das fichas de cães com paraplegia sem percepção à dor profunda, acometidos por DDIV toracolombar, submetidos à descompressão cirúrgica e encaminhados para a fisioterapia.

Foram incluídos, neste estudo, cães que realizaram fisioterapia em casa, mediante um protocolo fisioterapêutico domiciliar (GA) e aqueles efetuados em um centro especializado de fisioterapia (GB) em uma Instituição de ensino. Foram excluídos cães que vieram à morte por mielomalácia confirmada na necropsia e fichas clínicas incompletas.

Os protocolos de fisioterapia elaborados pelo Fisiovet compreenderam o uso de diferentes modalidades definidas de acordo com a evolução pós-operatória e a recuperação funcional do paciente. Inicialmente, todos os cães foram submetidos à aplicação da crioterapia, durante 72 horas de P0, sobre a incisão cirúrgica (Quadro 1).

O protocolo domiciliar (Quadro 1), orientado aos tutores, foi explicado detalhadamente no momento da alta médica que ocorreu após três dias de pós-operatório. Nas recomendações diárias estava a massagem da musculatura da coxa, deslizando os dedos ou a palma da mão em ritmo moderado e sincrônico; a movimentação passiva articular (MPA) dos membros pélvicos (MP) de todas as articulações simultaneamente, simulando o movimento de bicicleta; o estímulo do reflexo flexor dos MP, realizando pinçamento interdigital e resistência contra o movimento de flexão; a escovação dos coxins plantares; o posicionamento do animal em estação para sustentar o peso; fazer caminhadas com ou sem o auxílio da tipoia corporal em diferentes solos aderentes (grama, cimento, pedras, areia). Para os cães que iniciavam a deambulação, foi adicionada às recomendações diárias a caminhada sobre obstáculos e o subir e descer rampa (aclive e declive).

O protocolo fisioterapêutico realizado no Fisiovet (Quadro 1) iniciou com a massagem, MPA e reflexo flexor dos MP, executadas como mencionado anteriormente; alongamento em extensão e flexão; MPA de cada articulação dos MP; estimulação elétrica neuromuscular (EENM) de média frequência (Russa) sobre os músculos da coxa, conforme recomendações de Pelizzari et al. (2008).

A caminhada assistida com a tipoia corporal também foi utilizada até que os animais pudessem caminhar sozinhos. Para os que mantinham a sustentação dos membros pélvicos em estação, a plataforma proprioceptiva circular foi incluída. A hidroesteira, utilizada para estimular a movimentação dos MP, foi mantida com água ao nível do trocânter maior e variou o tempo de atividade de acordo com a melhora da resistência física de cada paciente.

Os animais que conseguiam dar passos eram estimulados a se movimentar sobre um colchão medindo três metros de comprimento. Esta atividade era realizada em forma de circuito, juntamente com o exercício de obstáculos, nos animais que caminhavam. A frequência e a duração do protocolo de fisioterapia foram definidas em três vezes por semana durante 90 dias de PO.

No período de novembro a dezembro de 2016 foram feitos contatos telefônicos com os tutores dos cães selecionados para a atualização de informações a respeito da recuperação funcional e da aplicação de um questionário sobre a QV (modificado de Mich \& Hellyer 2009) com no mínimo seis meses de PO. Para àqueles que não atendiam a primeira ligação, as tentativas foram realizadas durante uma semana, três vezes ao dia, em turnos diferentes, para só assim serem excluídos da lista. Foi solicitada a reavaliação clínica 
Quadro 1. Modalidades e atividades utilizadas nos protocolos dos cães paraplégicos sem dor profunda em decorrência da doença do disco intervertebral toracolombar após cirurgia descompressiva e submetidos à fisioterapia domiciliar ou em um centro especializado

\begin{tabular}{|c|c|c|c|}
\hline Modalidade/atividade & Tempo e/ou repetições & GA & GB \\
\hline Crioterapia & 20 minutos/a cada 3 horas & $\mathrm{X}$ & $\mathrm{X}$ \\
\hline Massagem de deslizamento & 5 minutos & $\mathrm{X}$ & $\mathrm{X}$ \\
\hline Alongamento & 20 segundos em cada posição & - & $\mathrm{X}$ \\
\hline MPA - Bicicleta & 30 vezes & $\mathrm{X}$ & $\mathrm{X}$ \\
\hline Reflexo flexor & 15 vezes & $\mathrm{X}$ & $\mathrm{X}$ \\
\hline Sustentação do peso em estação & 5 minutos & $\mathrm{X}$ & - \\
\hline Plataforma proprioceptiva circular & 2 a 3 minutos & - & $\mathrm{X}$ \\
\hline \multirow[t]{2}{*}{ Tipoia corporal } & 3 a 5 minutos & - & $\mathrm{X}$ \\
\hline & 5 a 10 minutos & $\mathrm{X}$ & - \\
\hline Caminhadas com guia & 5 a 10 minutos & $\mathrm{X}$ & - \\
\hline Rampa & 5 minutos & $\mathrm{X}$ & - \\
\hline
\end{tabular}

dos animais, mas, para os que não pudessem comparecer, um vídeo do cão se locomovendo.

O tutor respondeu itens de âmbito físico, comportamental e social (McMillan 2000), e por último, deu uma nota de 0 a 10 sobre a QV do cão, sendo classificada em excelente (nota 10), boa (notas 8 e 9), ruim (notas 5-7) e péssima (notas 0-4), conforme demonstrado na Figura 1.

Foram coletados dados a respeito da idade, sexo, raça, peso corporal, grau de disfunção neurológica decorridos 90 dias de PO, tempo para retorno à deambulação e desenvolvimento de reflexo de caminhar espinhal. Para os tutores que responderam o questionário perguntou-se sobre a recuperação funcional, presença de incontinência ou retenção urinária, tempo de sobrevida dos animais que vieram a óbito ou submetidos a eutanásia e nota atribuída à qualidade de vida PO.

A incontinência urinária foi considerada, neste estudo, quando o tutor respondia pelo menos uma das seguintes perguntas: se o cão urinava em locais inadequados (na própria cama), quando estava em movimento (andando) ou quando era pego no colo.

As deficiências neurológicas foram classificadas conforme Fingeroth \& Thomas (2015) em cinco graus. No grau I (GI) o animal apresentava somente dor, grau II (GII) paraparesia ambulatória, grau III (GIII) paraparesia não ambulatória, grau IV (GIV) paraplegia com presença de dor profunda e grau V (GV) a paraplegia com ausência de dor profunda.

$\mathrm{Na}$ análise estatística, para as variáveis quantitativas foram utilizadas média e desvio padrão e para as variáveis qualitativas, frequências absolutas e relativas. O software "Statistical Analysis System" (SAS), versão 9.02 realizou a análise comparativa de frequência, como o teste qui-quadrado para frequências cruzadas. Para comparação entre grupos, foi inicialmente aplicado um teste de normalidade (Shapiro Wilk) e em função da não-normalidade dos dados optou-se pelo teste Mann-Whitney (comparação de dois grupos). 0 nível de significância foi de $5 \%$.

Neste estudo, participaram 66 cães paraplégicos sem percepção a dor profunda acometidos por DDIV (Hansen tipo I) toracolombar. Vinte e oito cães foram submetidos ao protocolo domiciliar (GA) e 38 realizaram tratamento fisioterapêutico no centro especializado (GB).

Quanto às raças acometidas, 58\% (38/66) eram Dachshund, $21 \%(14 / 66)$ sem raça definida, $6 \%$ (4/66) poodle, 4,5\% (3/66) Yorkshire, 3\% (2/66) Lhasa Apso, 3\% (2/66) Cocker Spaniel, 3\% (2/66) Pequinês e 1,5\% (1/66) Basset Hound. A distribuição etária variou de 3 a 11 anos, com média de 5,8 anos, sendo 65,1\% (43/66) entre 3 e 6 anos e 33,3\% (22/66) entre 7 e 10 anos de idade. Em relação ao gênero, 36 eram fêmeas ( 15 castradas) e 30 machos (nove castrados), e a média de peso corporal foi de $8,1 \mathrm{~kg}$ (dois a $20,5 \mathrm{~kg})$, sendo $15 \%(10 / 66)$ menor que $5 \mathrm{~kg}, 62 \%$ (41/66) entre 5 e $10 \mathrm{~kg}$ e $16,6 \%(11 / 66)$ entre 10 e $15 \mathrm{~kg}$.

\section{RESULTADOS E DISCUSSÃO}

Neste estudo, foi verificada uma média de 28 sessões de fisioterapia nos cães de ambos os grupos, com duração de 69 e 67 dias de tratamento no GA e GB, respectivamente. Mesmo definindo a frequência e a duração nos protocolos fisioterapêuticos, nem sempre isso é possível de ocorrer. Os principais motivos estão relacionados à disponibilidade de tempo, custos, deslocamento dos pacientes, a melhora dos cães e o abandono do tratamento pelos tutores.

Hodgson et al. (2017) também encontraram variação nos tratamentos fisioterapêuticos indicados no pós-operatório de 87 cães com DDIV toracolombar em diferentes graus de lesão. Observaram intervalos de 3-66 dias na aplicação das modalidades, 12 a 238 modificações de protocolo, em período de uma a 40 semanas de acompanhamento. 
Questionário de avaliação da qualidade de vida pós-operatório

1) Após dias, meses ou anos da cirurgia (hoje, se seu animal está vivo):

a) ele consegue caminhar? Sim () Não ()

b) ele consegue movimentar as patas, mas não consegue caminhar? Sim () Não ()

c) ele não movimenta as patas? Sim, ele não movimenta ( ); Não, ele movimenta ()

d) quando a pata é apertada/ pinçada (teste de dor profunda realizado pelo veterinário) ele sente dor, grita, chora, tenta morder e/ou olha para a pata? Sim () Não ()

e) ele não tem dor profunda (é paraplégico) mas tem reflexos nas patas e por isso movimenta? Sim () Não ()

f) ele não tem dor profunda (è paraplégico) mas tem reflexos nas patas e por isso consegue caminhar (caminhar reflexo ou medular)? Sim () Não ()

2) Se seu animal consegue caminhar quanto tempo levou depois da cirurgia?_dias ( ), meses (), anos ().

3) Seu animal faz xixi normalmente? Sim () Não ()

4) Se seu animal não faz xixi normalmente, você faz a compressão da bexiga para ajudá-lo? Sim () Não ()

5) Seu animal faz xixi e em locais inadequados (ex.: na própria cama)? Sim () Não ()

6) Seu animal faz xixi quando está em movimento (andando) ou quando é pego no colo? Sim () Não ()

7) Você recebeu instruções no pós-operatório para realizar exercícios de fisioterapia em casa? Sim () Não ()

8) Você aplicou no seu animal exercícios de fisioterapia em casa? Sim () Não ()

9) Se você aplicou exercícios de fisioterapia em casa, qual foi a frequência semanal?_vezes por semana.

10) Se você aplicou a fisioterapia em casa, por quanto tempo depois da cirurgia?_dias ( ), meses ( ), anos( ).

11) Seu animal veio a óbito? Não () Sim (). Se sim, qual foi o motivo?

12) Seu animal foi submetido à eutanásia? Não () Sim (). Se sim, qual foi o motivo?

13) Se seu animal veio a óbito ou foi submetido à eutanásia, quanto tempo depois da cirurgia?_dias (), meses

(), anos ().

14) Domínio do seu animal de estimação

a) Físico:

O nivel de atividade está normal? Sempre () Frequentemente () Raramente () Nunca ()

É capaz de completar tarefas normais cotidianas (brincar, subir escadas, trabalhar, etc.)? Sempre ()

Frequentemente () Raramente () Nunca ()

Está comendo quantidade normal? Sempre () Frequentemente () Raramente () Nunca ()

Se levanta para saudá-lo? Sempre () Frequentemente () Raramente () Nunca ()

Corre ao redor da casa? Sempre () Frequentemente () Raramente () Nunca ()

Consegue se levantar com facilidade? Sempre () Frequentemente () Raramente () Nunca ()

Consegue se posicionar normalmente para fazer as necessidades? Sempre () Frequentemente () Raramente ()

Nunca ()

Está respirando normalmente? Sempre () Frequentemente () Raramente () Nunca ()

Deixa você tocar a área dolorosa ou da cirurgia? Sempre () Frequentemente () Raramente () Nunca ()

b) Comportamental:

Está se comportando normalmente? Sempre () Frequentemente () Raramente () Nunca ()

Cuida da própria aparência? Sempre () Frequentemente () Raramente () Nunca ()

Dorme confortavelmente? Sempre () Frequentemente () Raramente () Nunca ()

Está urinando e defecando normalmente? Sempre () Frequentemente () Raramente () Nunca ()

Parece deprimido? Sempre () Frequentemente () Raramente () Nunca ()

Está ansioso ou nervoso? Sempre () Frequentemente () Raramente () Nunca ()

Chora inesperadamente? Sempre () Frequentemente () Raramente () Nunca ()

Está agressivo? Sempre () Frequentemente () Raramente () Nunca ()

Está reagindo adversamente a alguma medicação? Sempre () Frequentemente () Raramente () Nunca ()

Está feliz? Sempre () Frequentemente () Raramente () Nunca ()

c) Social:

Fica feliz ao ver você chegar em casa? Sempre () Frequentemente () Raramente () Nunca ()

Interage normalmente com você e outras pessoas? Sempre () Frequentemente () Raramente () Nunca ()

Interage normalmente com outros animais? Sempre () Frequentemente () Raramente () Nunca ()

15) De 0 a 10, qual a qualidade de vida do seu animal?

16) Comente aspectos da vida do seu animal que você considera importante depois da cirurgia de hérnia de disco:

Fig.1. Questionário sobre a recuperação funcional e qualidade de vida dos cães paraplégicos sem percepção a dor profunda acometidos por DDIV toracolombar e submetidos ao protocolo de fisioterapia domiciliar ou em um centro especializado (modificado de Mich \& Hellyer 2009). 
Do total de cães (GA e GB), 53\% (35/66) permaneceram em GV, 42,4\% (28/66) evoluíram para GII e 4,6\% (3/66 - um GA e dois $\mathrm{GB}$ ) recuperaram a dor profunda, mas não a habilidade de caminhar independente (deficiência neurológica GIII). Trinta e um animais (47\%) recuperaram a habilidade de caminhar no período de até 90 dias de PO (média de 39 dias/desvio padrão $\pm 22,7$ dias). Destes, três $(4,6 \%)$ desenvolveram o reflexo de caminhar espinhal (média de 68 dias) e outros três (um GA e dois GB) após esse período, com média de 354 dias. Não houve diferença quanto ao protocolo domiciliar ou em um centro especializado no desenvolvimento do reflexo de andar espinhal (Quadros 2 e 3).

Dos cães que evoluíram para GII, 43\% (12/28) eram do GA e $42 \%(16 / 38)$ do GB, apresentando recuperação na média de 40 (desvio padrão $\pm 28,2$ dias) e 36 dias (desvio padrão $\pm 17,6$ dias), respectivamente. Não houve diferença entre os grupos estudados. Um cão do GA e dois do GB desenvolveram reflexo de caminhar espinhal em até 90 dias de PO. Vale ressaltar que, embora a fisioterapia possa ser considerada uma terapia adjuvante indispensável na recuperação de cães com DDIV após descompressão cirúrgica, não se recomenda utilizá-la como primeira opção para casos aqui estudados, exceto para aquelas situações em que o paciente não possa ser submetido ao tratamento cirúrgico.
Na avaliação de 31 cães paraplégicos, com ausência de percepção a dor profunda e submetidos a um programa de reabilitação domiciliar realizado por Hodgson et al. (2017), pode-se observar que 74\% tornaram-se ambulatórios após a cirurgia. Os autores destacaram que destes cães, $68 \%$ resultaram em deambulação sem alterações neurológicas perceptíveis ou paraparesia ambulatória com ataxia leve, demonstrando que as chances de uma recuperação completa foram maiores quando comparados com um grupo controle.

No entanto, cães com deficiências neurológicas severas no pré-operatório, sinais clínicos graves ou com recuperação lenta estão propensos a participar de um programa de fisioterapia realizado por terapeuta específico da área, podendo subestimar a eficiência das modalidades. Estes foram os motivos relatados por Hodgson et al. (2017) quando observaram que o tratamento fisioterapêutico instituído não foi capaz de acelerar a recuperação dos animais.

Observando os mesmos parâmetros de recuperação em publicações que utilizaram alguma modalidade para reabilitação, Kazakos et al. (2005) obtiveram 25\% de recuperação satisfatória em uma média de 37 dias de $\mathrm{PO}$ e no momento da alta médica recomendaram aos tutores a massagem, exercícios passivos e ativos. Jeffery et al. (2016) relataram 58\% de recuperação funcional em um intervalo médio de 49 dias, utilizando nos

Quadro 2. Informações dos 25 cães paraplégicos sem percepção a dor profunda com doença do disco intervertebral toracolombar-após cirurgia descompressiva e submetidos à fisioterapia domiciliar

\begin{tabular}{|c|c|c|c|c|c|c|c|c|c|c|c|c|}
\hline $\mathrm{n}$ & Grau ciru & Grau PO 1 & RC 1 & CE 1 & Grau PO 2 & CE 2 & RC 2 & $\mathrm{IU}$ & Eutanásia & Morte & $\mathrm{TS}$ & Nota QV \\
\hline 1 & GV & $\mathrm{GV}$ & 90 & Sim & $\mathrm{GV}$ & Sim & NA & Sim & Não & Não & NA & 9 \\
\hline 2 & GV & GV & NA & Não & GV & Sim & 180 & Sim & Não & Não & NA & 6 \\
\hline 3 & GV & GV & NA & Não & GV & Não & NA & Sim & Não & Não & NA & 7 \\
\hline 4 & GV & GV & NA & Não & GV & Não & NA & Sim & Não & Não & NA & 10 \\
\hline 5 & GV & GV & NA & Não & GV & Não & NA & Sim & Não & Não & NA & 8 \\
\hline 6 & GV & GV & NA & Não & GV & Não & NA & Sim & Não & Não & NA & 8 \\
\hline 7 & GV & GV & NA & Não & GV & Não & NA & Sim & Sim & Sim & 150 & 5 \\
\hline 8 & GV & GV & NA & Não & GV & Não & NA & Sim & Sim & Sim & 90 & 0 \\
\hline 9 & GV & GV & NA & Não & GV & Não & NA & Sim & Sim & Sim & 300 & 4 \\
\hline 10 & GV & GV & NA & Não & GV & Não & NA & Sim & Sim & Sim & 90 & 4 \\
\hline 11 & $\mathrm{GV}$ & $\mathrm{GV}$ & NA & Não & $\mathrm{GV}$ & Não & NA & Sim & Não & Sim & 365 & 3 \\
\hline 12 & GV & GV & NA & Não & GV & Não & NA & Sim & Não & Não & NA & 7 \\
\hline 13 & GV & GV & NA & Não & $\mathrm{GV}$ & Não & NA & Sim & Não & Não & NA & 10 \\
\hline 14 & GV & GIII & NA & NA & GIII & NA & NA & Sim & Não & Não & NA & 7 \\
\hline 15 & GV & GII & 75 & NA & GII & NA & NA & Sim & Não & Não & NA & 9 \\
\hline 16 & GV & GII & 90 & NA & GII & NA & NA & Sim & Não & Não & NA & 7 \\
\hline 17 & GV & GII & 45 & NA & GII & NA & NA & Sim & Não & Não & NA & 8 \\
\hline 18 & GV & GII & 30 & NA & GII & NA & NA & Não & Não & Não & NA & 10 \\
\hline 19 & GV & GII & 60 & NA & GII & NA & NA & Não & Não & Não & NA & 10 \\
\hline 20 & GV & GII & 30 & NA & GII & NA & NA & Não & Não & Não & NA & 10 \\
\hline 21 & GV & GII & 21 & NA & GII & NA & NA & Sim & Não & Não & NA & 10 \\
\hline 22 & GV & GII & 15 & NA & GII & NA & NA & Não & Não & Não & NA & 10 \\
\hline 23 & GV & GII & 30 & NA & GII & NA & NA & Sim & Não & Não & NA & 7 \\
\hline 24 & GV & GII & 7 & NA & GII & NA & NA & Sim & Não & Sim & 30 & 5 \\
\hline 25 & GV & GII & 21 & NA & GII & NA & NA & Sim & Não & Não & NA & 7 \\
\hline
\end{tabular}

Dados obtidos dos tutores de no mínimo seis meses de pós-operatório, Grau ciru= grau de deficiência neurológica na cirurgia, PO = pós-operatório, Grau PO 1 = grau de deficiência neurológica até 90 dias de PO, RC 1 = tempo de retorno da caminhada até 90 dias de PO, CE 1 = desenvolvimento do caminhar espinhal até 90 dias de PO, Grau PO 2 = grau de deficiência neurológica no momento do contato com tutor, RC 2 = tempo de retorno da caminhada após 90 dias de PO, CE 2 = desenvolvimento do caminhar espinhal após 90 dias de PO, IU = incontinência urinária, TS = tempo de sobrevida, $\mathrm{QV}=$ qualidade de vida, $\mathrm{NA}=$ não se aplica. 
Quadro 3. Informações dos 26 cães paraplégicos sem percepção a dor profunda com doença do disco intervertebral toracolombar após cirurgia descompressiva e submetidos à fisioterapia em centro especializado

\begin{tabular}{|c|c|c|c|c|c|c|c|c|c|c|c|c|}
\hline $\mathrm{n}$ & Grau ciru & Grau PO 1 & $\mathrm{RC} 1$ & CE 1 & Grau PO 2 & CE 2 & $\mathrm{RC} 2$ & $\mathrm{IU}$ & Eutanásia & Morte & TS & Nota QV \\
\hline 1 & GV & $\mathrm{GV}$ & 85 & $\mathrm{Sim}$ & GV & Sim & NA & Não & Não & Não & NA & 8 \\
\hline 2 & GV & GV & 30 & Sim & GV & Sim & NA & Não & Não & Não & NA & 10 \\
\hline 3 & GV & GV & NA & Não & GV & Sim & 730 & Sim & Não & Não & NA & 9 \\
\hline 5 & GV & GV & NA & Não & GV & Não & NA & Sim & Não & Não & NA & 8 \\
\hline 6 & GV & GV & NA & Não & GV & Não & NA & Sim & Não & Não & NA & 7 \\
\hline 9 & GV & GV & NA & Não & GV & Não & NA & Sim & Não & Não & NA & 7 \\
\hline 10 & GV & GV & NA & Não & GV & Não & NA & Sim & Não & Não & NA & 10 \\
\hline 11 & GV & GV & NA & Não & GV & Não & NA & Sim & Não & Não & NA & 10 \\
\hline 12 & GV & GV & NA & Não & GV & Não & NA & Sim & Não & Não & NA & 8 \\
\hline 13 & GV & GV & NA & Não & GV & Não & NA & Sim & Não & Sim & 730 & 5 \\
\hline 18 & GV & GII & 43 & NA & GII & NA & NA & Sim & Não & Sim & 240 & 8 \\
\hline 19 & GV & GII & 34 & NA & GII & NA & NA & Sim & Não & Não & NA & 9 \\
\hline 20 & GV & GII & 45 & NA & GII & NA & NA & Sim & Não & Sim & 1095 & 10 \\
\hline 21 & GV & GII & 32 & NA & GII & NA & NA & Sim & Não & Não & NA & 10 \\
\hline 22 & GV & GII & 26 & NA & GII & NA & NA & Sim & Não & Não & NA & 8 \\
\hline 23 & $\mathrm{GV}$ & GII & 31 & NA & GII & NA & NA & Não & Não & Não & NA & 10 \\
\hline 24 & GV & GII & 20 & NA & GII & NA & NA & Não & Não & Não & NA & 10 \\
\hline 25 & GV & GII & 16 & NA & GII & NA & NA & Sim & Não & Não & NA & 10 \\
\hline 26 & GV & GII & 10 & NA & GII & NA & NA & Sim & Não & Não & NA & 10 \\
\hline
\end{tabular}

Dados obtidos dos tutores de no mínimo seis meses de pós-operatório, Grau ciru = grau de deficiência neurológica na cirurgia, PO = pós-operatório, Grau PO 1 = grau de deficiência neurológica até 90 dias de PO, RC 1 = tempo de retorno da caminhada até 90 dias de PO, CE $1=$ desenvolvimento do caminhar espinhal até 90 dias de PO, Grau PO 2 = grau de deficiência neurológica no momento do contato com tutor, RC $2=$ tempo de retorno da caminhada após 90 dias de PO, CE 2 = desenvolvimento do caminhar espinhal após 90 dias de PO, IU = incontinência urinária, TS = tempo de sobrevida, $\mathrm{QV}=$ qualidade de vida, $\mathrm{NA}=$ não se aplica.

cuidados PO a fisioterapia adaptada para cada paciente. Ainda, Arias et al. (2007) encontraram médias de 62,5\% em 53 dias e relataram o uso da movimentação passiva e da tipoia corporal no pós-operatório. Apesar de citarem a fisioterapia nos tratamentos, os autores supracitados não discutiram a influência da fisioterapia e comparação de protocolos durante o período de avaliação.

Ao analisar estes dados, nota-se uma variação nos resultados de recuperação funcional para cães paraplégicos sem percepção a dor profunda e submetidos a cirurgia descompressiva, tornando-se um fator complicante no momento de instituir o prognóstico. Mesmo em trabalhos que não citaram a fisioterapia como adjuvante ao tratamento, as taxas de recuperação e média de tempo são muito variadas, como 25\% em 12 dias de pós-operatório (Festugatto et al. 2008), 41,3\% em 12 meses (Laitinen \& Puerto 2005), 45,5\% entre 12 e 20 semanas (Muguet-Chanoit et al. 2012), $53 \%$ em 12 semanas (Olby et al. 2003), 62\% em 2 semanas (Scott \& McKee 1999) e 73,3\% em 27 dias (Santos et al. 2012).

Quanto ao questionário sobre a qualidade de vida PO dos animais (Fig.1), pode-se verificar, nos Quadros 2 e 3, que ambos os grupos apresentaram índices de incontinência urinária de
84\% (graus II, III e V). Dos cães que recuperaram para grau II, $63,6 \%(7 / 11)$ eram do GA e $80 \%$ (8/10) do GB. Os valores revelados neste estudo estão acima dos encontrados na literatura, com 4,1\% (Festugatto et al. 2008) e 32\% (Olby et al. 2003). Estes autores ainda afirmaram que a incontinência urinária estava presente em todos os pacientes em grau V, diferente deste estudo, em que dois cães que desenvolveram o caminhar espinhal possuíam controle da micção.

Quanto ao tempo de sobrevida dos animais que vieram a óbito ou que foram submetidos à eutanásia no pós-operatório, houve diferença entre os grupos $(\mathrm{p}<0,05)$, sendo em média 171 dias no GA (n=6) e 546 dias no GB $(n=6)$. Outro dado observado foi a maior tendência a eutanásias nos cães do $\mathrm{GA}(\mathrm{n}=4)$ quando comparado aos do $\mathrm{GB}(\mathrm{n}=0)(\mathrm{p}=0,0506)$.

Quando são necessários determinados cuidados de enfermagem ao longo da vida do animal, estes podem ser aceitáveis para alguns tutores, mas para outros, impossível de se manter (Wall 2015). A constante visita a um serviço especializado de fisioterapia foi importante não só por auxiliar na recuperação do paciente, mas provavelmente por estimular e orientar o tutor na manutenção e na melhoria da $\mathrm{QV}$, principalmente para aqueles que não tiveram recuperação 
completa. Estas constatações também foram relatadas por Hodgson et al. (2017), com índices de complicações significativamente maiores nos animais do grupo controle (sem fisioterapia).

Quanto as notas atribuídas pelos tutores à qualidade de vida PO, ficaram na média de 7 (ruim) no GA e 8 (boa) no GB, mas nos animais em GV foi de 6 (ruim) no GA e 8 (boa) no GB. Quando classificada em QV excelente, boa, ruim ou péssima, observamos, respectivamente, para o GA 28\%, 20\%, 36\% e 16\%; e para o GB 38,5\%, 38,5\%, 23\% e 0\% (Fig.2).

As razões para as notas abaixo de 10 atribuídas pelos tutores, foram a incontinência urinária dos animais (56\%, 19/34), dificuldade de locomoção (53\%, 18/34), lesões cutâneas abrasivas $(18 \%, 6 / 34)$, infecções urinárias recorrentes $(6 \%, 3 / 34)$ e auto-mutilação $(3 \%, 1 / 34)$, cuja nota atribuída para QV foi zero. Laitinen \& Puerto (2005) também relataram como problemas pós-operatório a infecção urinária e auto-mutilação, respectivamente em 6,5\% e 4\%. Bauer et al. (1992) avaliaram os problemas relatados por tutores de cães paraplégicos e os resultados foram semelhantes, mas destacaram que todos os tutores discordaram sobre seus cães terem uma QV ruim, 84\% afirmaram que o comportamento estava tão bom quanto antes da cirurgia e 74\% acreditavam que seus cães não percebiam que estavam paraplégicos.

O método para avaliação da QV deste trabalho, apesar de subjetivo, leva em consideração a opinião do tutor frente a nova condição do seu animal, fazendo um comparativo com a vida antes e depois da DDIV. Pode-se perceber que os animais que permaneceram em grau $\mathrm{V}$ e submetidos ao tratamento fisioterapêutico em centro especializado (GB), em média, foram avaliados com uma nota de QV classificada boa, ou seja, o tutor não considerou o fato de o animal não caminhar com os membros pélvicos como determinante para diminuir sua QV. As perguntas de âmbito físico, comportamental e social precederam a nota mensurada pelo tutor, que não teve acesso à classificação atribuída a cada uma delas. Com isso, pôde-se evitar uma possível influência negativa pela denominação péssima e ruim, deixando mais fidedigna a pontuação com a real impressão do mesmo.

Levine et al. (2007), em um estudo retrospectivo, também utilizaram um questionário para avaliar o sucesso do tratamento conservativo em cães com diagnóstico presuntivo de DDIV toracolombar, incluindo um escore numérico de 0 a 10 para a QV dos animais. Afirmaram que as respostas baseadas na

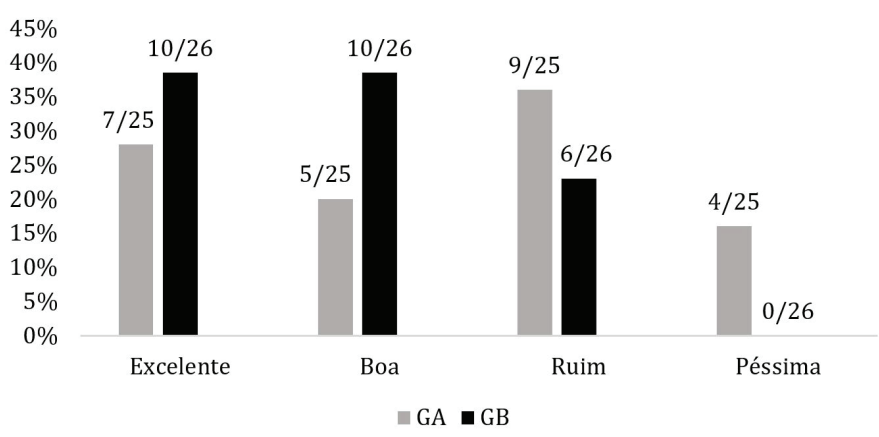

Fig.2. Classificação das notas atribuída à qualidade de vida dos cães submetidos à fisioterapia domiciliar (GA) ou em um centro especializado (GB) de acordo com a percepção dos tutores. impressão do tutor têm a vantagem de estarem mais bem respaldados para determinar um nível de deficiência e de QV aceitável. Um questionário específico para cães com lesões medulares foi desenvolvido e aplicado, sendo adicionada a Escala Visual Analógica (EVA) como forma de avaliação para $\mathrm{QV}$, obtendo uma correlação positiva significante quando comparada com outros métodos objetivos (Budke et al. 2008, Levine et al. 2008). Além destes, outros estudos em medicina veterinária utilizaram EVA para quantificar a QV e em geral tiveram alta confiabilidade (McMillan 2000, Mullan \& Main 2007).

Além disso, assim como apontado por Levine et al. (2007), uma abordagem relativamente simplista foi escolhida devido à natureza subjetiva dos resultados. 0 questionário utilizado foi projetado especificamente para coletar dados simples sobre a situação clínica final e a QV dos animais, de acordo com a percepção do tutor, podendo ocorrer falhas nos resultados que para serem validados necessitam de um desenho prospectivo.

Uma das limitações deste trabalho foi a ausência de um grupo controle, ou seja, sem fisioterapia, para demonstrar a influência deste tratamento na recuperação dos animais. Outro fator que pode ter influenciado nos resultados foi o tempo de até oito anos para contato com os tutores. Logo, sugerem-se novos estudos prospectivos, com a formação de um grupo controle, que permitam comprovar a importância da fisioterapia na recuperação funcional de cães com DDIV toracolombar após descompressão cirúrgica da medula espinhal.

Neste estudo, não houve diferença quanto a recuperação funcional dos cães entre os grupos submetidos a fisioterapia domiciliar ou em um centro especializado. Mesmo assim, notou-se que os cães GB (centro especializado) foram considerados com uma QV pós-operatória melhor, provavelmente pelo fato de poderem ser monitorados constantemente pelo fisioterapeuta em busca de complicações oriundas da doença, bem como orientar os tutores a melhor forma de manejar os animais nestas condições. Bauer et al. (1992) já haviam relatado a importância do veterinário em informar, conscientizar e dar suporte aos tutores de cães paraplégicos, proporcionando a satisfação dos mesmos em relação a sua própria QV e de seu cão.

\section{CONCLUSÕES}

De acordo com os resultados obtidos, pode-se verificar que não houve diferença nas taxas e tempo de recuperação funcional de cães paraplégicos sem percepção a dor profunda em decorrência de DDIV toracolombar após descompressão cirúrgica, submetidos a um protocolo de fisioterapia em um centro especializado ou em domicílio.

A avaliação do tempo de sobrevida dos cães que evoluíram para a morte, decisão pela eutanásia e nota atribuída à QV demonstraram que os animais que realizaram tratamento fisioterapêutico em centro especializado provavelmente foram beneficiados pelo estreito acompanhamento e orientação aos tutores até 90 dias de pós-operatório.

\section{REFERÊNCIAS}

Arias M.V.B., Nishioka C.M., Garcia C.O., Reia A.Z., Baraúva Júnior D. \& Marcasso R.A. 2007. Avaliação dos resultados clínicos após cirurgia descompressiva em cães com doença de disco intervertebral. Arq. Bras. Med. Vet. Zootec. 59:1445-1450. 
Bauer M., Glickman N., Glickman L., Toombs J., Golden S. \& Skowronek C. 1992. Follow-up study of owner attitudes toward home care of paraplegic dogs. J. Am. Vet. Med. Assoc. 200(12):1809-1816. <PMid:1639682>

Brisson B.A. 2010. Intervertebral disc disease in dogs. Vet. Clin. N. Am., Small Anim. Pract. 40(5):829-858. <PMid:20732594>

Budke C.M., Levine J.M., Kerwin S.C., Levine G.J., Hettlich B.F. \& Slater M.R. 2008. Evaluation of a questionnaire for obtaining owner-perceived, weighted quality-of-life assessments for dogs with spinal cord injuries. J. Am. Vet. Med. Assoc. 233(6):925-930. <PMid:18795853>

Dewey C.W. \& Costa R.C. 2016. Practical Guide to Canine and Feline Neurology. 3rd ed. Wiley Blackwell, New Delhi. 672p.

Festugatto R., Mazzanti A., Raiser A.G., Pelizzari C., Salbego F.Z., Beckmann D.V., Pereira D.T. \& Santos R.P. 2008. Recuperação funcional de cães com doença do disco intervertebral toracolombar submetidos ao tratamento cirúrgico. Ciência Rural 38(8):2232-2238.

Fingeroth J.M. \& Thomas W.B. 2015. Advances in Intervertebral Disc Disease in Dogs and Cats. Wiley-Blackwell, Iowa. 321p.

Granger N. \& Carwardine D. 2014. Acute spinal cord injury tetraplegia and paraplegia in small animals. Vet. Clin. N. Am., Small Anim. Pract. 44(6):11311156. <PMid:25441629>

Hodgson M.M., Bevan J.M., Evans R.B. \& Johnson T.I. 2017. Influence of in-house rehabilitation on the postoperative outcome of dogs with intervertebral disk herniation. Vet. Surg. 46(4):566-573. <PMid:28233909>

Jeffery N.D., Barker A.K., Hu H.Z., Alcott C.J., Kraus K.H., Scanlin E.M., Granger N. \& Levine J.M. 2016. Factors associated with recovery from paraplegia in dogs with loss of pain perception in the pelvic limbs following intervertebral disk herniation. J. Am. Vet. Med. Assoc. 248(4):386-394. <PMid:26829270>

Kazakos G., Polizopoulou Z.S., Patsikas M.N., Tsimopoulos G., Roubies N. \& Dessiris A. 2005. Duration and severity of clinical signs as prognostic indicators in 30 dogs with thoracolumbar disk disease after surgical decompression. J. Vet. Med. 52(3):147-152. <PMid:15836447>

Laitinen O.M. \& Puerto D.A. 2005. Surgical decompression in dogs with thoracolumbar intervertebral disc disease and loss of deep pain perception: a retrospective study of 46 cases. Acta Vet. Scand. 46(1/2):79-85. $<$ PMid:16108215>

Levine J.M., Levine G.J., Johnson S.I., Kerwin S.C., Hettlich B.F. \& Fosgate G.T. 2007. Evaluation of the success of medical management for presumptive thoracolumbarintervertebral disk herniation in dogs. Vet. Surg. 36(5):482491. <PMid:17614930>

Levine J.M., Budke C.M., Levine G.J., Kerwin S.C., Hettlich B.F. \& Slater M.R. 2008 Owner-perceived, weighted quality-of-life assessments in dogs with spinal cord injuries. J. Am. Vet. Med. Assoc. 233(6):931-935. <PMid:18795855>

McMillan F.D. 2000. Quality of life in animals. J. Am. Vet. Med. Assoc. 216(12):1904-1910. <PMid:10863585>

Mich P.M. \& Hellyer P.W. 2009. Métodos objetivos e categóricos para avaliar a dor e analgesia, p.78-109. In: Gaynor J.S. \& Muir W.W (Eds), Manual de Controle da Dor. $2^{\underline{a}}$ ed. MedVet, São Paulo.

Muguet-Chanoit A.C., Olby N.J., Lim J.H., Gallagher R., Niman Z., Dillard S., Campbell J., Early P., Mariani C.L., Muñana K.R., Freeman C., Platt S.R., Kent M., Giovanella C. \& Longshore R.C. 2012. The cutaneous trunci muscle reflex: a predictor of recovery in dogs with acute thoracolumbar myelopathies caused by intervertebral disc extrusions. Vet. Surg. 41(2):200206. <PMid:22150443>

Mullan S. \& Main D. 2007. Preliminary evaluation of a quality-of-life screening programme for pet dogs. J. Small Anim. Pract. 48(6):314-322. <PMid:17490443>

Olby N., Levine J., Harris T., Muñana K., Skeen T. \& Sharp N. 2003. Long-term functional outcome of dogs with severe injuries of the thoracolumbar spinal cord: 87 cases (1996-2001). J. Am. Vet. Med. Assoc. 222(6):762769. <PMid:12675299>

Pelizzari C., Mazzanti A., Raiser A.G., Lopes S.T.A., Graça D.L., Salbego F.Z., Ramos A.T., Festigatto R., Beckmann D.V., Cunha M.M., Santos R.P., Cargnelutti J.F., Pereira D.T. \& Martins T.B. 2008. Estimulação elétrica neuromuscular de média frequência (russa) em cães com atrofia muscular induzida. Ciência Rural 38(3):738-742.

Santos R.P., Beckmann D.V., Aiello G., Berté L., Ripplinger A., Polidoro Neto D. \& Mazzanti A. 2012. Recuperação funcional de cães paraplégicos com doença do disco intervertebral toracolombar sem percepção à dor profunda submetidos ao tratamento cirúrgico: 15 casos (2006-2010). Pesq. Vet. Bras. 32(3):243-246.

Scott H.W. \& McKee W.M. 1999. Laminectomy for 34 dogs with thoracolumbar intervertebral disc disease and loss of deep pain perception. J. Small Anim. Pract. 40(9):417-422. <PMid:10516947>

Wall R. 2015. Physical rehabilitation for the paralyzed patient, p.279-286. In: Fingeroth J.M. \& Thomas W.B. (Eds), Advances in Intervertebral Disc Disease in Dogs and Cats. Wiley-Blackwell, Iowa. 